\title{
Ochratoxin A Causes DNA Damage and Cytogenetic Effects but No DNA Adducts in Rats
}

\author{
Angela Mally ${ }^{\dagger}$ Gaetano Pepe $^{\ddagger}$ Srivani Ravoori, ${ }^{\S}$ Mario Fiore,$\ddagger$ \\ Ramesh C. Gupta, ${ }^{\S}$ Wolfgang Dekant, ${ }^{\dagger} * *$ and Pasquale Mosesso
}

\begin{abstract}
Department of Toxicology, University of Würzburg, Germany, Dipartimento di Agrobiologia e Agrochimica, Università degli Studi della Tuscia, Viterbo, Italy, and Pharmacology \& Toxicology/ Brown Cancer Center, University of Louisville, Louisville, Kentucky
\end{abstract}

Received December 16, 2004

\begin{abstract}
Ochratoxin A (OTA) is a potent nephrotoxin and renal carcinogen in rats, but the mechanism of OTA tumorigenicity is unknown. Ochratoxin A has been shown to be negative in many genetic toxicology test in vitro. However, the potential of OTA to induce genotoxic effects has not been investigated in male rats, the most sensitive species for OTA-induced tumor formation. In this study, male F344 rats were repeatedly administered OTA $(0,250,500,1000$, and 2000 $\mu \mathrm{g} / \mathrm{kg}$ of body wt) or the non-chlorinated analogue ochratoxin B (OTB; $2000 \mu \mathrm{g} / \mathrm{kg}$ of body wt) for 2 weeks (5 days/week), and DNA breakage was analyzed in target and nontarget tissues using the comet assay both in the absence and presence of formamidopyrimidine-DNA (Fpg) glycosylase. Potential DNA-adduct formation was also analyzed in the target organ kidney by ${ }^{32} \mathrm{P}$-postlabeling using two different solvent systems. DNA-strand breaks were evident in liver, kidney, and spleen of animals treated with OTA, and a similar degree of DNA damage was observed in rats treated with OTB, despite the lower toxicity of OTB. Moreover, the presence of DNA damage did not correlate with histopathological alterations, which were evident in the kidney but not in the liver. In liver and kidney, the extent of DNA damage was further enhanced in the presence of Fpg glycosylase, which is known to convert oxidative DNA damage into strand breaks, suggesting the presence of oxidative DNA damage. Oxidative DNA damage as a mechanism of OTA-dependent DNA damage is consistent with the absence of lipophilic DNA adducts as assessed by ${ }^{32} \mathrm{P}$-postlabeling analysis. No spots indicative of OTA-related DNA adducts were observed in kidney DNA extracted from OTA-treated animals by ${ }^{32} \mathrm{P}$-postlabeling analysis, despite the use of synthetic standard for postulated adducts. A small, but not significant, increase in the incidence of chromosomal aberrations (essentially chromatid and chromosome-type deletions) was observed in splenocytes from rats treated with OTA in vivo and subsequently cultured in vitro to express chromosomal damage. These aberrations are also compatible with oxidative DNA lesions since they are not typically caused by chemical carcinogens which form covalent DNA adducts. Together, with the lack of evidence for formation of lipophilic DNA adducts as assessed by postlabeling, these data suggest that OTA may cause genetic damage in both target and nontarget tissues independent of direct covalent binding to DNA.
\end{abstract}

\section{Introduction}

The mycotoxin and food contaminant ochratoxin A (OTA, ${ }^{1}$ Chart 1) is one of the most potent renal carcinogens studied by the National Cancer Institute/National Toxicology Program (NCI/NTP) to date. Little is known regarding the mechanism of tumor formation by OTA, and the genotoxic activity of OTA has been assessed in a variety of standard genetic toxicology tests. In general, only weak genotoxic effects have been observed, and the contribution of genotoxicity to renal tumor formation by

* Author for correspondence: Prof. Dr. Wolfgang Dekant, Department of Toxicology, University of Würzburg, Versbacher Str. 9, 97078 Würzburg, Germany. Tel: +49-931-20148449. Fax: +49-931-20148865

E-mail: dekant@toxi.uni-wuerzburg.de.

$\dagger$ University of Würzburg.

¥ Università degli Studi della Tuscia.

$\S$ University of Louisville.

${ }^{1}$ Abbreviations: OTA, ochratoxin A; OTB, ochratoxin B; Fpg, formamidopyrimidine-DNA glycosylase; FCS, fetal calf serum; 8-OHdG, 8-oxo-7,8-dihydro-2'-deoxyguanosine; dGMP, 2'-deoxyguanosine3'-monophosphate.

\section{Chart 1}

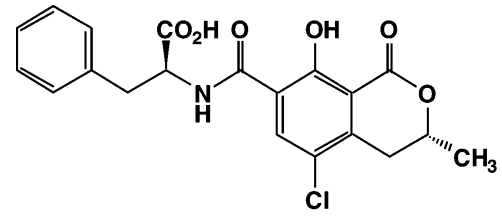

Ochratoxin A

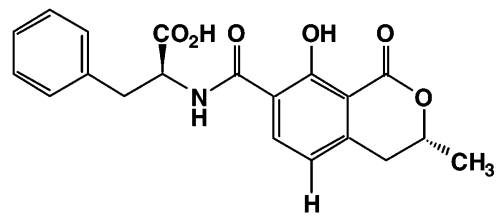

Ochratoxin B

OTA remains unknown. Numerous studies have shown that OTA is not mutagenic in Salmonella typhimurium both in the presence and absence of metabolic activation 